\title{
TV/Series
}

$10 \mid 2016$

Guerres en séries (II)

\section{La Seconde Guerre mondiale dans Band of Brothers (HBO, 2001) et The Pacific (HBO, 2010)}

\section{Guillaume Piketty}

\section{(2) OpenEdition}

1 Journals

Édition électronique

URL : http://journals.openedition.org/tvseries/1897

DOI : 10.4000/tvseries.1897

ISSN : 2266-0909

Éditeur

GRIC - Groupe de recherche Identités et Cultures

Référence électronique

Guillaume Piketty, "La Seconde Guerre mondiale dans Band of Brothers (HBO, 2001) et The Pacific (HBO, 2010)»,TV/Series [En ligne], 10 | 2016, mis en ligne le 01 décembre 2016, consulté le 19 avril 2019. URL : http://journals.openedition.org/tvseries/1897 ; DOI : 10.4000/tvseries.1897

Ce document a été généré automatiquement le 19 avril 2019

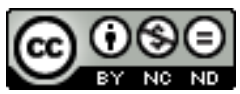

TV/Series est mis à disposition selon les termes de la licence Creative Commons Attribution - Pas d'Utilisation Commerciale - Pas de Modification 4.0 International. 


\title{
La Seconde Guerre mondiale dans Band of Brothers (HBO, 2001) et The Pacific (HBO, 2010)
}

\author{
Guillaume Piketty
}

1 Réalisées à quelque dix années d'intervalle, les mini-séries Band of Brothers (2001) et The Pacific (2010) se veulent l'une et l'autre à mille lieues de tout fantasme de guerre ${ }^{1}$. Toutes deux projettent en effet de représenter et de donner à comprendre la Seconde Guerre mondiale telle qu'elle fut réellement vécue par certains combattants américains, en Europe de l'ouest pour la première puis sur le front Pacifique pour la seconde. Force est de constater qu'elles y parviennent avec un succès variable. Ces mini-séries présentent un certain nombre de similitudes. Toutes deux postérieures à Saving Private Ryan (1998), elles sont inscrites dans une forme de filiation avec le film de Steven Spielberg. Mises en chantier et diffusées par HBO, elles ont été produites par les sociétés Playtone ${ }^{2}$ et Dreamworks. Band of Brothers a pour producteurs exécutifs Tom Hanks et Steven Spielberg, et pour coproducteurs exécutifs Stephen E. Ambrose, Gary Goetzman et Tony To. Hanks, Spielberg et Goetzman ont été producteurs exécutifs de The Pacific, tandis que Tony To, Eugene Kelly, Graham Yost et Bruce C. Mc Kenna en étaient les coproducteurs exécutifs. En d'autres termes, les concepteurs des deux mini-séries sont sensiblement les mêmes. Par ailleurs, quelques-uns des scénaristes et des réalisateurs d'épisodes de Band of Brothers ont travaillé pour The Pacific. Dans l'un et l'autre cas, d'excellents acteurs ont été sélectionnés, mais aucune star ne figure au casting.

2 Chacune des mini-séries est organisée en dix épisodes. Band of Brothers accompagne les soldats de la Easy Company du 506 ème régiment de la $101^{\text {ème }}$ division aéroportée (les «Screaming Eagles») depuis leur formation militaire dans le camp Toccoa en Géorgie, aux États-Unis, jusqu'au 15 août $1945^{3}$ en Autriche, en passant notamment par la Normandie, l'opération Market Garden, le siège de Bastogne et l'Allemagne envahie. The Pacific évoque quant à elle le versant américano-japonais du conflit. Elle se concentre en particulier sur les destins de trois combattants appartenant à différents régiments de la 1 ère division de Marines : John Basilone, choisi en raison de ses hauts faits et de la publicité 
qui leur fut accordée durant la guerre, Robert Leckie et Eugene Sledge, sélectionnés, eux, à cause de la très grande qualité de leurs mémoires ${ }^{4}$. La mini-série évoque notamment les batailles de Guadalcanal (7 août 1942 - 9 février 1943), Cape Gloucester (26 décembre 1943 - 22 avril 1944), Peleliu (15 septembre - 25 novembre 1944), Iwo Jima (19 février - 26 mars 1945) et Okinawa (1er avril - 21 juin 1945). Dans les deux cas et bien qu'il s'agisse de combats terrestres, l'armée de terre américaine (US Army) est donc presque totalement $a^{a b s e n t e}{ }^{5}$. En d'autres termes, sont présentées des troupes d'élite censées préparer le terrain aux gros bataillons des forces terrestres, des troupes qui portent les premiers coups à l'ennemi ou bien débloquent une situation difficile, voire compromise.

Les deux séries veulent placer le spectateur " au ras du champ de bataille », donner à voir et à ressentir ce qu'a pu être l'expérience des combattants. Elles s'affranchissent par conséquent de toute référence aux enjeux diplomatiques, économiques, sociaux ou même de politique intérieure dont la Seconde Guerre mondiale a pourtant été riche. Elles ont été l'une et l'autre saluées par la critique et récompensées ${ }^{6}$, et ont connu un solide succès public ${ }^{7}$. Pourtant, Band of Brothers et The Pacific diffèrent singulièrement par leur propos. Ces différences sont notamment sensibles dans la présentation qui est faite des protagonistes, des modalités de combat et des premiers temps de la « sortie de guerre ». Elles tiennent à la nature des sources employées, au type de combat livré, aux influences historiographiques et cinématographiques subies, ainsi qu'aux évolutions des contextes politique américain et international lors de la première décennie du XXI ${ }^{e}$ siècle.

\section{Des « héros » de plus en plus désabusés}

4 D'une mini-série à l'autre, le positionnement et le comportement des personnages principaux évoluent substantiellement. Si l'entraînement des hommes de la Easy Company est raconté par le menu tout au long du premier épisode de Band of Brothers, celui des Marines, en revanche, est à peu près passé sous silence. Après avoir consacré quelques instants à la décision prise par Leckie (James Badge Dale) de rejoindre l'US Marine Corps (USMC) juste après Pearl Harbor, The Pacific présente Basilone (Jon Seda) écoutant avec ses camarades le déjà mythique colonel Chesty Puller ${ }^{8}$ (William Sadler) et la vaine tentative faite par Sledge (Joseph Mazello) pour s'engager, puis nous entraîne par une ellipse à Guadalcanal. Par la suite, quelques allusions sont faites à l'entraînement de Leckie, quelques plans sont proposés de celui subi par Sledge, et le sergent-chef Basilone est brièvement montré en action alors qu'il est devenu instructeur'. Plus largement, les protagonistes des deux séries sont des combattants d'élite ou... supposés tels. Les parachutistes de la Easy Company ne sont bien évidemment pas exempts de faiblesses, mais ils s'affirment comme d'excellents combattants, loyaux, soudés. Ils forment une belle illustration $\mathrm{du}$ " groupe primaire ${ }^{10}$ " à la fois en termes de relations entre soldats et d'efficacité sur le terrain. Parmi les bonus de l'édition DVD britannique publiée en 2010, la série propose un documentaire d'1h20 dont le titre même, We Stand Alone Together, insiste sur les vertus de la fraternité d'armes et renvoie notamment au mythe profondément américain du "Last Stand ${ }^{11}$ ", si possible victorieux comme à Bastogne en décembre 1944. Dans Band of Brothers, les blessés n'ont de cesse de rejoindre le front au plus vite pour soutenir leurs camarades. Quant aux nouvelles recrues, les «remplacements» ( replacements), elles finissent pour la plupart d'entre elles par s'en sortir plutôt correctement. Côté Pacifique, les Marines sont bien sûr de très efficaces soldats. Mais ils ne sont certainement pas des machines à combattre, et accessoirement à tuer. À travers 
eux, les "fatigues de la guerre", pour reprendre l'expression d'Arlette Farge ${ }^{12}$, sont nettement et régulièrement montrées. Souvent abattus par l'ennemi à peine arrivés, voire délibérément sacrifiés par les vétérans afin que ceux-ci puissent sauver leurs propres vies, les remplacements subissent un sort décidément peu enviable ${ }^{13}$.

Compétente, digne de confiance et rarement battue en brèche dans Band of Brothers, la hiérarchie est régulièrement critiquée, voire brocardée, dans The Pacific. Certes, au héros parachutiste Winters ${ }^{14}$ (Damian Lewis) répond le capitaine des Marines Andrew «Ack Ack » Haldane (Scott Gibson), le «bienaimé commandant de compagnie » évoqué par Sledge dans la dédicace de ses mémoires de guerre, qui tombe à Bloody Nose Ridge (Peleliu). En d'autres termes, la figure paternelle du commandant de compagnie existe dans l'une et l'autre série ${ }^{15}$. Mais les similitudes s'arrêtent là. Dans Band of Brothers, un mauvais officier tel que le premier commandant de la compagnie, le capitaine Sobel (David Schwimmer), est de facto démis de ses fonctions dès l'épisode 1. Le patron du régiment est lointain et critiquable, mais les officiers subalternes sont pour la plupart de grande qualité, y compris le troublant lieutenant Speirs (Matthew Settle) dont on ne saura jamais s'il a massacré ou non un groupe de prisonniers de guerre (épisode 2). Quand, à Bastogne, l'unité se trouve à nouveau affligée d'un pitoyable chef, le lieutenant Dike (Peter $\left.O^{\prime} M e a r a^{16}\right)$, celui-ci est très vite remplacé par l'excellent et très courageux ${ }^{17}$ Speirs (épisode 7).

Ce média ne peut être affiché ici. Veuillez vous reporter à l'édition en ligne http:// journals.openedition.org/tvseries/1897

Le sergent-chef Lipton (Donnie Wahlberg) est nommé sous-lieutenant sur le champ de bataille (épisode 7) : s'il commence par nourrir quelques doutes sur sa capacité à assumer de telles fonctions, il fait face à ses nouvelles responsabilités et connaît le succès. C'est d'ailleurs lui, force du symbole, qui reçoit la reddition du général allemand (épisode 10).

Ce média ne peut être affiché ici. Veuillez vous reporter à l'édition en ligne http:// journals.openedition.org/tvseries/1897

Enfin, quand le Major Winters désobéit aux ordres (épisode 8), c'est pour de bonnes et évidentes raisons, et son insubordination n'est ni remarquée ni, donc, punie.

Le tableau proposé par The Pacific est tout autre. Bien sûr, le personnage haut en couleur du très compétent et courageux colonel Chesty Puller est mis en avant. Mais, le capitaine Haldane mis à part, les officiers subalternes apparaissent comme peu utiles et, souvent, peu dignes de confiance ${ }^{18}$. John Basilone reçoit la Médaille d'honneur ${ }^{19}$ pour sa conduite à Guadalcanal sans être vraiment sûr de la mériter davantage que d'autres. Le vieux sergent-chef Haney (Gary Sweet), solide, expérimenté et en apparence indestructible, finit par craquer. À l'instar de Leckie ou bien de Sledge et son ami Phillips (Ashton Holmes), les simples Marines passent très vite maitres ès critique de la hiérarchie. Soulignons enfin la présence constante du merveilleux personnage de $S n a f u^{20}$, i.e. le soldat puis caporal Merriel Shelton (Rami Malek). Par sa seule existence et son surnom, celui-ci dit beaucoup: la désillusion, la volonté de survivre à tout prix, la perte de certaines valeurs, la solitude morale qui en découle, etc. Est-il besoin de préciser qu'un tel personnage n'existe pas dans Band of Brothers? En d'autres termes, au pur héros Winters, répondent les anti-héros désabusés Leckie, Sledge et même Basilone qui, après avoir reçu la Médaille d'honneur, se trouve très vite embrigadé pour la promotion des War Bonds ${ }^{21}$, 
profite de la belle vie, et finalement retourne au combat pour se faire presque immédiatement tuer (épisode 8).

Enfin, les relations entre combattants et civils sont bien davantage mises en évidence dans The Pacific que dans Band of Brothers. Cette différence entre les deux mini-séries est d'autant plus frappante que le système de permissions était infiniment moins pratique, efficace et confortable dans le Pacifique qu'en Europe. Dans Band of Brothers, les relations entre les parachutistes de la Easy Company et les civils britanniques avant le D-Day puis après la bataille de Normandie sont brièvement montrées (épisodes 1 et 3). Il en est de même pour les interactions avec les Hollandais dans Eindhoven libéré au cours de l'opération Market Garden, puis avec les réfugiés belges et luxembourgeois que la rapidité de la progression allemande a conduits à se réfugier à Bastogne en décembre 1944. Une fois le Rhin franchi, des civils allemands épuisés, défaits et passablement hébétés sont rapidement mis en scène. Mais l'essentiel de la série n'est décidément pas là. Tout autre est la perspective proposée par The Pacific. Pensons par exemple à la scène poignante durant laquelle les Marines éreintés rescapés de Guadalcanal découvrent que leur combat a fait l'objet d'un véritable « marketing » aux USA ${ }^{22}$.

Ce média ne peut être affiché ici. Veuillez vous reporter à l'édition en ligne http://

Pensons également au repos australien après Guadalcanal qui fait l'objet de l'épisode 3 tout entier, aux tournées effectuées par Basilone aux États-Unis afin de promouvoir l'achat de War Bonds, ou encore au dernier épisode exclusivement consacré au retour et sur lequel nous reviendrons. Aussi le Home Front est-il un personnage à part entière de la seconde série, mais un personnage dont, soulignons-le, le décalage avec les combattants apparait de plus en plus flagrant au fil des épisodes.

\section{L'enfer du combat et ses conséquences}

D'une œuvre à l'autre, les représentations de l'ennemi, du combat et de ses conséquences évoluent substantiellement. Band of Brothers met en scène un ennemi allemand redoutable de maîtrise et d'expérience, endurci par des années de guerre. Mais cet ennemi est rarement montré de près. Surtout, il n'est peu ou prou dévalorisé qu'en toute fin de série. Ainsi, par exemple, les massacres qui ont été perpétrés par certaines unités allemandes au cours de la bataille des Ardennes ${ }^{23}$ ne sont pas évoqués. Ce n'est que dans l'épisode 9 , centré sur la découverte des camps de concentration nazis, que sont abordées les raisons profondes du conflit, et plus largement la notion de "Greatest Generation ${ }^{24}$ » américaine engagée dans une "guerre juste». Toutefois, cette découverte des camps de la mort est en quelque sorte compensée par la magnifique scène du dernier épisode au cours de laquelle le général allemand adresse un discours d'adieu à ses hommes : en filigrane, la délicate question est ainsi posée de savoir si ces vétérans épuisés de la Wehrmacht sont vraiment si différents de leurs homologues américains.

11 Pour sa part, The Pacific montre un ennemi à plusieurs facettes. Dans les premiers épisodes, le soldat japonais est régulièrement évoqué comme un combattant hors pair, une sorte de surhomme, terrifiant d'efficacité, de résistance et d'abnégation. 
Ce média ne peut être affiché ici. Veuillez vous reporter à l'édition en ligne http://

Il arrive bien sûr que cet ennemi devienne un homme qui souffre et qui meurt, un opposant humanisé confronté lui aussi à la peur et au malheur ${ }^{25}$. Mais, globalement, cet adversaire demeure assez largement incompréhensible. Sa capacité de survivre durablement dans des conditions extrêmes est une preuve de son " anormalité ». Illustré par ses charges suicidaires ${ }^{26}$ ou par sa volonté de ne se rendre à aucun prix, son code d'honneur ne fait pas sens aux yeux des Marines. En d'autres termes, la série met en scène une incompréhension américaine croissante adossée à de profondes différences culturelles. Cette incompréhension aboutit souvent à une animalisation de l'ennemi japonais ${ }^{27}$, voire à des prises de trophées humain ${ }^{28}$. Celles-ci sont montrées sans pudeur aucune qu'il s'agisse par exemple de couper des oreilles ou encore de prélever des dents en or sur des soldats nippons qui, de toute évidence, ne sont pas complètement morts (épisode 7).

Ce média ne peut être affiché ici. Veuillez vous reporter à l'édition en ligne http:// journals.openedition.org/tvseries/1897

Enfin, The Pacific met soigneusement en scène un autre ennemi, qui devient par moment le véritable adversaire, celui dont il faut absolument se défier et qu'il convient parfois de combattre : l'environnement... Bien sûr, la pénibilité du combat dans les haies du bocage normand ou dans le froid, la neige et la glace des Ardennes est montrée dans Band of Brothers. Mais ces conditions de (sur)vie n'ont rien à voir avec la chaleur et l'humidité de Guadalcanal, l'enfer de la jungle de Cape Gloucester, la chaleur écrasante de Peleliu, ou encore les pluies torrentielles et la boue d'Okinawa. À cet égard, l'épisode 4 de The Pacific, qui se déroule à Cape Gloucester, à l'extrême-ouest de l'île de Nouvelle-Bretagne, ellemême située au large de la Nouvelle-Guinée, est particulièrement éloquent. L'environnement y est dépeint comme la principale menace, encore accentuée par le fait que les Japonais peuvent à tout moment surgir pour frapper, avant de replonger dans les profondeurs de la jungle. Une tension permanente et une véritable anxiété en découlent qui, peu à peu, usent les Marines.

On l'a dit, les deux mini-séries sont les héritières de Saving Private Ryan et, notamment, de sa longue et célèbre scène d'ouverture décrivant l'assaut sur Omaha Beach au matin du 6 juin 1944. Dans les deux cas, la violence de guerre est donc explicitement montrée. Mais elle l'est selon des formes et à des degrés variables. Dans Band of Brothers, elle est justifiée militairement et relativement contenue. Ainsi, par exemple, la conquête de la batterie allemande menaçant Utah Beach par une petite escouade placée sous les ordres de Winters devient une sorte de ballet bien réglé débouchant sur un authentique succès (épisode 2). Il en est de même de la campagne de Normandie dans son ensemble, malgré les largages initiaux de parachutistes largement ratés, les considérables difficultés créées par les haies du bocage, et la résistance acharnée des Allemands. L'opération Market Garden et la bataille des Ardennes laissent une impression similaire même si la première fut sanglante et aboutit à un échec, et si la seconde, dans Bastogne assiégé (21-26 décembre 1944) notamment, soumit régulièrement les combattants à une très rude épreuve. Dans The Pacific, au contraire, la violence présentée à l'écran devient presque affolante : démence des combats nocturnes à Guadalcanal, absurdité de la lutte à Cape 
Gloucester, débarquement à Peleliu et horreur absolue sur l'aéroport ${ }^{29}$, lance-flammes en action un peu partout, enfer de pluie, de boue et de saleté à Okinawa où, de surcroît, il arrive que les civils soient transformés en bombes ambulantes... La guerre dans le Pacifique est ainsi montrée pour ce qu'elle fut : un véritable « laminoir de l'âme ${ }^{30}$ ».

Fort logiquement, les moments de la blessure, physique ou psychique, et, éventuellement, de la mort sont bien plus crûment mis en scène dans The Pacific que dans Band of Brothers. Bien sûr, des parachutistes souffrent et tombent. Mais la raison de leur blessure ou de leur mort est connue : être touché ainsi, quelquefois mortellement, a malgré tout du sens. En cas de blessure, ces hommes sont la plupart du temps secourus vite et bien. Et ce, même à Bastogne où pourtant les Américains manquent de tout ${ }^{31}$. À cet égard, le personnage du « Medic » Eugene Roe (Shane Taylor) auquel est largement consacré le 6 ème épisode est emblématique ${ }^{32}$. Combien différente est The Pacific... Les débarquements y virent régulièrement à la boucherie. Certaines blessures atroces, ou simplement reçues dans des circonstances presque aberrantes ${ }^{33}$, sont précisément montrées. Ceux qui, tels Sledge et Snafu, s'en sortent sans une égratignure ne comprennent pas par quel miracle ils y sont parvenus, soulignant implicitement l'absurdité de la guerre. Absurdes également, certaines morts pour des objectifs qui n'en valaient pas la peine ou dans des circonstances qui auraient pu être évitées.

Soulignons enfin que les traumatismes psychiques, certes présents dans Band of Brothers, le sont infiniment plus dans The Pacific: au soldat Albert Blithe (Marc Warren) temporairement aveugle, au Medic Eugene Roe, et à l'épisode 7 notamment consacré au «Breaking Point» du lieutenant Compton (Neal McDonough), répondent les survivants hagards de Guadalcanal (épisode 2), l'énurésie de Leckie, le suicide du Marine Lebec qui n'en peut plus d'être trempé nuit et jour par une pluie incessante (épisode 4), le « capitaine minuit» («Captain Midnight») Stanfield qui marche la nuit dans la chambrée d'hôpital à Banika en se croyant aux commandes de son avion (épisode 4), ou encore la rupture du vieux et jusque-là inébranlable sergent-chef Haney. D'une œuvre à l'autre, les combats sont toujours plus horribles et marqués par la peur et la souffrance, le malheur et le traumatisme, ainsi que par un fort sentiment d'absurdité et de vanité.

Enfin, les conséquences du conflit sur les combattants et, plus largement, le délicat processus de "sortie de guerre " sont incomparablement mieux évoqués dans The Pacific que dans Band of Brothers. Celle-ci s'achève en effet par un dernier épisode autrichien assez bucolique. Les suites du conflit ne sont évoquées par la voix off du Major Winters que sous la forme d'un rapide « Que sont-ils devenus? » qui neutralise totalement l'enjeu, pourtant complexe, redoutable et redouté, de reprise au long cours d'une vie de temps de paix. Tout autre est la dixième et dernière partie de The Pacific. Cet ultime épisode concentre en effet de façon subtile un grand nombre des défis qui, à la fin d'un conflit, attendent celles et ceux, civils et militaires, combattants ou non, qui ont fait l'expérience du phénomène guerrier. D'entrée, les scènes dans le train mettent en évidence la douloureuse séparation d'avec les camarades de combat. Un peu plus loin, Leckie et Sledge vivent de passablement troublantes retrouvailles avec des proches qui, du fait de la guerre, ne le sont plus vraiment. La magnifique et poignante scène au cours de laquelle Lena Basilone (Annie Parisse) rencontre ses beaux-parents et son beau-frère pour la première fois ${ }^{34}$ illustre les souffrances du deuil.

19 L'économie morale de la reconnaissance, c'est-à-dire ces gestes, médailles, pensions, défilés et commémorations par lesquels une société reconnaît, et tente de compenser, le sacrifice de celles et ceux qu'elle a envoyés au combat ${ }^{35}$, est régulièrement évoquée, qu'il 
s'agisse de la Médaille d'honneur de John Basilone que Lena remet à ses beaux-parents, des rubans de décoration sur les uniformes, ou encore des égards rendus à certains vétérans. Présente aussi, on l'a dit, la plus ou moins profonde incompréhension par la société civile de ce qui s'est réellement passé dans les îles du Pacifique. Ainsi, le jour de la victoire sur le Japon, dans l'hôpital où Leckie et ses camarades blessés poursuivent leur convalescence, seuls les civils se réjouissent; les anciens combattants, eux, sont simplement tristes et abattus, incapables de la moindre exubérance. Les différences de comportement entre le père et la mère de Sledge sont également éloquentes : lui, qui a servi comme médecin en Europe sur les champs de bataille de la Première Guerre mondiale, mesure ce que son fils doit affronter; elle est tout simplement perdue. La jeune et jolie Vera ${ }^{36}$ (Caroline Dhavernas) achève de conquérir Robert Leckie car elle comprend et respecte ses difficultés à trouver les mots, voire ses silences. Telle n'est en revanche pas le cas des membres de la famille de Leckie auxquels celui-ci finit par indiquer, provoquant, qu'avec ses camarades il s'est essentiellement battu "pour (avoir) la télévision ».

Les difficultés de la réinsertion, estudiantine ou professionnelle, sont précisément mises en évidence par le truchement de la candidature de Sledge à l'Alabama Polytechnic Institute ${ }^{37}$.

Ce média ne peut être affiché ici. Veuillez vous reporter à l'édition en ligne http:// journals.openedition.org/tvseries/1897

Enfin, le syndrome de stress post-traumatique (« Post-Traumatic Stress Disorders (PTSD) ») est nettement décrit via les personnages de Leckie, de Phillips et de Sledge. Les symptômes manifestés par ce dernier, en particulier, sont illustrés par le menu: cauchemars nocturnes, difficultés à participer à des festivités, incapacité d'utiliser à nouveau une arme à feu pour chasser, comportement souvent aboulique, etc.

\section{Conclusion : des mini-séries sous influences}

Ainsi, la représentation du phénomène guerrier proposée par The Pacific est nettement plus complète que celle offerte par Band of Brothers. Quatre faisceaux d'éléments sont à l'origine de cette évolution. Tout d'abord, les deux séries ont été fondées sur des sources de nature différente. Band of Brothers fut écrite principalement à partir du best-seller éponyme de l'historien Stephen E. Ambrose ${ }^{38}$. Celui-ci, qui fut également conseiller historique des scénaristes, travaillait avec grand sérieux à partir de témoignages et d'archives. Mais il lui était manifestement difficile de se départir d'une certaine admiration pour les vétérans de la Easy Company et, plus généralement, pour les anciens combattants américains de la Seconde Guerre mondiale. En outre, au début de sa carrière, cet historien prolifique était tombé sous le charme d'Eisenhower dont il était devenu le biographe. Difficile pour lui de critiquer les décisions et les actions du Commandant en chef (Supreme Commander)... Il a donc produit une histoire de facture assez classique. Le scénario de The Pacific a été composé, on l'a dit, à partir des remarquables mémoires de Robert Leckie ${ }^{39}$ et de Eugene Sledge ${ }^{40}$. Les souvenirs de Chuck Tatum qui combattit au côté de John Basilone ${ }^{41}$, d'autres mémoires ${ }^{42}$, et des documents intimes ou privés ont été également utilisés ${ }^{43}$. En d'autres termes, la mini-série est d'abord fondée sur les récits et les objets personnels des acteurs eux-mêmes ou de leurs proches ${ }^{44}$. 

scène un combat relativement classique qui a pour théâtre l'Europe de l'ouest. On est loin, très loin, des déserts d'Afrique de l'Est ${ }^{45}$ et du Nord, des montagnes yougoslaves ou des immensités est-européennes. Si la guerre a bien évidemment constitué un choc terrible pour les soldats, dont elle a traumatisé un certain nombre, la nature du combat et le lieu où celui-ci s'est déroulé ne leur ont pas porté des coups supplémentaires. Tout autre fut la lutte dans le Pacifique. Là, outre le terrible ennemi japonais, les Marines ont dû affronter la jungle et l'humidité, des terrains arides et rocailleux, une chaleur souvent écrasante ou des pluies torrentielles, une vie animale particulièrement inquiétante, etc. Ce contexte guerrier bien spécifique a participé pleinement du bouleversement vécu par les combattants. C'est d'ailleurs ce que l'ex-parachutiste devenu chauffeur de taxi confie à Leckie dans le dernier épisode de The Pacific : à ses yeux, et même s'il notamment connu les terribles combats de Normandie, la vraie guerre a bien été livrée par les «GI-Rines ${ }^{46}$ » dans le Pacifique.

En troisième lieu, les scénaristes et les réalisateurs d'épisode de The Pacific ont subi de nouvelles influences historiographiques et cinématographiques. Rappelons-le, les concepteurs de Band of Brothers se sont fondés sur une historiographie de facture classique. The Pacific, en revanche, porte la marque des études plus récentes et modernes du phénomène guerrier, en particulier fondées sur l'anthropologie historique. Ces travaux donnent notamment toute leur place aux techniques de combat et à l'environnement, aux relations entre combattants et aux émotions, aux blessures, aux soignants et à la mort, aux civils et à l'arrière (« Home Front»), aux représentations, etc. Autant d'éléments que l'on retrouve dans les tableaux qui sont proposés de la guerre livrée par les Marines. Par ailleurs, on l'a dit, The Pacific s'inscrit dans la suite de Flags of our Fathers et de Letters from Iwo Jima. Mais la mini-série subit également l'influence d'œuvres telles que, par exemple, Black-Hawk Down (Ridley Scott, 2001) et sa violence épouvantable, Combat Diary: The Marines of Lima Company (Michael Epstein, 2006) et son souci de regarder la guerre avec les yeux des combattants ${ }^{47}$, ou encore l'émouvant Taking Chance (Ross Katz, 2009) qui montre la puissance du travail de deuil, individuel et collectif, au sein d'une société américaine profondément meurtrie par la guerre en Irak et en Afghanistan.

Enfin, les contextes politiques américain et, plus largement, international ont évidemment imprimé leurs marques sur les deux mini-séries. Band of Brothers fut mise en chantier et tournée avant le 11 septembre 2001, alors que le "gendarme du monde » ne s'était pas encore engagé en Irak et en Afghanistan. The Pacific, pour sa part, porte l'empreinte de ces deux conflits: leur violence épouvantable; leur absurdité; les mensonges sur la base desquels ils furent déclenchés ; l'idée que la guerre n'est peut-être pas toujours la solution et devrait, dans l'idéal, être mise hors-la-loi ; le malheur, enfin, que l'événement guerrier apporte, à titre individuel, aux combattants et à leurs familles. Bien que tournée à la toute fin du $\mathrm{XX}^{\mathrm{e}}$ siècle, Band of Brothers est ainsi la lointaine héritière de certaines superproductions des années 1960 et $1970^{48}$. Avec, sous-jacente, l'idée de "Greatest Generation" ", i.e. la génération de la Grande Dépression qui a tout surmonté et a fini par remporter la dernière guerre « juste » de l'histoire des États-Unis, le second conflit mondial. En refusant de considérer comme négligeables des éléments tels que, par exemple, l'environnement, les émotions ${ }^{50}$ et les traumatismes psychiques, les relations entre soldats et civils, ou encore le processus de sortie de guerre, The Pacific 
s'inscrit, elle, résolument dans l'après-Vietnam et ses syndromes de stress posttraumatique, l'après-Irak et l'après-Afghanistan.

\section{BIBLIOGRAPHIE}

AMBrose Hugh, The Pacific. Hell was an Ocean Away, New York, New American Library, 2010.

AMBROSE Stephen E., Band of Brothers, E Company, 506th Regiment, 101st Airborne from Normandy to Hitler's Eagle's Nest, New York, Simon \& Schuster, 1992

BROKAW Tom, The Greatest Generation, New York, Random House, 1998.

CABANES Bruno, «La démobilisation des soldats français », Les cahiers de la paix, $\mathrm{n}^{\circ}$ 7, Nancy, Presses de l'Université de Nancy, 2000, p. 55-65.

CABANES Bruno, La victoire endeuillée. La sortie de guerre des soldats français (1918-1920), Paris, Seuil, 2004.

DOWER John, War without Mercy. Race and Power in the Pacific War, New York, Pantheon Books, 1987. FARGE Arlette, Les fatigues de la guerre, Paris, Gallimard, 1996.

FUSSELL Paul, Wartime : Understanding and Behaviour in the Second World War, New York - Oxford, Oxford University Press, 1989.

FUSSELL Paul, The Boys' Crusade. American G.I.s in Europe : Chaos and Fear in World War Two, Londres, Phoenix, 2005.

GRAY Jessie Glenn, The Warriors. Reflexions on Men in Battle, New York, Harcourt Brace, 1959. Reed., Lincoln et Londres, University of Nebraska Press, 1998 ; trad. fr. Tallandier, 2012

HAMNER christopher H., Enduring Battle. American Soldiers in Three Wars, 1776-1945, Lawrence, University Press of Kansas, 2011.

HARRISON Simon, «Skull trophies of the Pacific War : Transgressive objects of remembrance », Journal of the Royal Anthropological Institute, December 2006, 12, p. 817-836

LECKIE Robert, Helmet for my Pillow. From Parris Island to the Pacific, New York, Bantam Books, 2010 (1957).

MARSHALL S.L.A., Men against Fire : The Problem of Battle Command, Norman, University of Oklahoma Press, 2000.

PHILLIPS sydney, You'll Be Sor-ree! A Guadalcanal Marine Remembers the Pacific War, New York, Berkley Trade, 2012.

PIKETTY Guillaume, La bataille des Ardennes. 16 décembre 1944-31 janvier 1945, Paris, Tallandier, 2013. Rééd. collection « Texto », 2015.

PIKETTY Guillaume et TROUPLIN vladimir, Les compagnons de l'aube. Archives inédites des compagnons de la Libération, Paris, Éditions Textuel, 2014. 
ROSE Kenneth D., Myth and The Greatest Generation. A Social History of Americans in World War II, New York, Taylor and Francis Group, 2008.

SCHRIJVERS Peter, The GI War Against Japan. American Soldier in Asia and the Pacific During World War II, New York, New York University Press, 2002.

SHILS Edward A. et JANOWITZ Morris, « Cohesion and Disintegration in the Wehrmacht in World War II », The Public Opinion Quarterly, vol. 12, n 2 (Summer 1948), p. 280-315.

SLEDGE Eugene B., With The Old Breed. At Peleliu and Okinawa, New York, Ballantine Books, 2010.

SLEDGE Eugene B., China Marine. An Infantryman's Life After World War Two, New York, Oxford

University Press, 2003.

STOUFFER samuel et al., The American Soldier: Combat and its Aftermath (Studies in Social Psychology in World Ward II, vol. 2), Princeton, Princeton University Press, 1949.

TATUM chuck, Red Blood, Black Sand. Fighting alongside John Basilone from Boot Camp to Iwo Jima, New York, Berkley Trade, 2013.

\section{ANNEXES}

\section{Filmographie}

Le jour le plus long (Ken Annakin et alii, 1962)

La grande évasion (John Sturges, 1963)

Tora! Tora! Tora! (Richard Fleischer, Kinji Fukasaku et Toshio Masuda, 1970)

Patton (Franklin Schaffner, 1970)

La bataille de Midway (Jack Smight, 1976)

Black-Hawk Down (Ridley Scott, 2001)

Flags of our Fathers (Clint Eastwood, 2006)

Letters from Iwo Jima (Clint Eastwood, 2006)

Combat Diary: The Marines of Lima Company (Michael Epstein, 2006)

Taking Chance (Ross Katz, 2009)

\section{NOTES}

1. Pour reprendre le titre donné à la partie du colloque dans laquelle a été présentée la communication dont le présent texte est issu : « Fantasmer la Seconde Guerre mondiale ».

2. Fondée en 1996 par Tom Hanks et le producteur Gary Goetzman.

3. Jour de la capitulation du Japon.

4. Robert Leckie, Helmet for my Pillow. From Parris Island to the Pacific, New York, Bantam Books, 2010 (1957) ; Eugene B. Sledge, With The Old Breed. At Peleliu and Okinawa, New York, Ballantine Books, 2010 (très vite après sa première parution, en 1981, ce livre est devenu un bestseller).

5. Et bien sûr aussi la Navy et l'Air Force (laquelle, à cette époque, fait partie de l'Army). 
6. Nommée à plus de 25 reprises, Band of Brothers a remporté plus de 20 récompenses dont le Golden Globe et l'Emmy Award de la meilleure mini-série en 2002 ; nommée quelque 23 fois, The Pacific a remporté 14 récompenses, dont l'Emmy Award de la meilleure mini-série en 2010.

7. Plus net cependant pour Band of Brothers dont, pour leur première diffusion aux États-Unis, les épisodes ont rassemblé entre 5 et 10 millions de téléspectateurs alors que l'audience de The Pacific n'a, elle, pas dépassé les 3 millions.

8. Le patron du $7^{\mathrm{ème}}$ régiment de Marines dont, dans l'épisode de la série, le discours est une sorte d'écho à celui prononcé par le général Patton au tout début du film éponyme de Franklin Schaffner (1970).

9. Boot camp.

10. Edward A. Shils et Morris Janowitz, «Cohesion and Disintegration in the Wehrmacht in World War II ", The Pubic Opinion Quarterly, vol. 12, n 2 (Summer 1948), pp. 280-315. S.L.A. Marshall, Men against Fire: The Problem of Battle Command, Norman, University of Oklahoma Press, 2000. Samuel Stouffer et al., The American Soldier: Combat and its Aftermath (Studies in Social Psychology in World Ward II, vol. 2), Princeton, Princeton University Press, 1949. Pour une récente et féconde discussion de cette notion, voir par exemple Christopher H. Hamner, Enduring Battle. American Soldiers in Three Wars, 1776-1945, Lawrence, University Press of Kansas, 2011.

11. Par exemple à Fort Alamo ou à Little Big Horn.

12. Arlette Farge, Les fatigues de la guerre, Paris, Gallimard, 1996.

13. Sur cette question, voir notamment: Paul Fussell, Wartime: Understanding and Behaviour in the Second World War, New York - Oxford, Oxford University Press, 1989; Paul Fussell, The Boys' Crusade. American G.I.s in Europe: Chaos and Fear in World War Two, Londres, Phoenix, 2005 ; Jessie Glenn Gray, The Warriors. Reflexions on Men in Battle, New York, Harcourt Brace, 1959. Reed., Lincoln et Londres, University of Nebraska Press, 1998 ; trad. fr. Tallandier, 2012.

14. Qui a néanmoins une sorte de «double» et ami, plus tourmenté, passablement alcoolique, éventuellement faillible mais très intelligent, le capitaine Dixon.

15. "Andrew Haldane wasn't an idol, he was the company ", écrit notamment Sledge.

16. «Qui n'est pas mauvais car il prend de mauvaises décisions mais parce qu'il ne prend pas de décision ", commente l'un de ses subordonnés.

17. Pour preuve, notamment, son incroyable course au beau milieu du dispositif ennemi à Foy, près de Bastogne, afin de débloquer une situation singulièrement compromise (épisode 7).

18. Dans l'épisode 4, l'un d'eux se permet même de priver l'un de ses hommes (Leckie) d'une de ses prises de guerre (un coffre japonais).

19. Qui est, rappelons-le, la plus haute distinction militaire américaine.

20. Pour « Situation Normal All Fucked Up ».

21. Écho au film Flags of our Fathers de Clint Eastwood (2006).

22. "You are heroes back home ".

23. Voir par exemple Guillaume Piketty, La bataille des Ardennes. 16 décembre 1944-31 janvier 1945, Paris, Tallandier, 2013. Rééd. collection « Texto », 2015.

24. Tom Brokaw, The Greatest Generation, New York, Random House, 1998.

25. Écho au film Letters from Iwo Jima de Clint Eastwood (2006).

26. Surnommées «banzaï charges » par les Américains.

27. Sur ces questions, on se reportera avec profit à : John Dower, War without Mercy. Race and Power in the Pacific War, New York, Pantheon Books, 1987 ; Peter Schrijvers, The GI War Against Japan. American Soldier in Asia and the Pacific During World War II, New York, New York University Press, 2002.

28. Voir à nouveau, John Dower, War without Mercy... Op. cit. et Peter Schrijvers, The GI War Against Japan... Op. cit. Voir aussi Simon Harrison, «Skull trophies of the Pacific War: Transgressive objects of remembrance ", Journal of the Royal Anthropological Institute, December 2006, 12, pp. 817-836. 
29. Avec notamment cette course à découvert qui n'a rien à envier aux sorties de tranchées de la Grande Guerre pour se faire hacher menu par les mitrailleuses japonaises.

30. Pour reprendre une expression gaullienne à propos de la bataille de Verdun - Témoignage de Pierre Messmer à l'auteur, 1999.

31. L'épisode 6 montre toutefois que les soins ne débouchent pas toujours mais néanmoins l'effort et l'infrastructure sont là.

32. Et aussi celui de la nurse qui meurt à la fin de l'épisode.

33. Comme celle de Leckie à Peleliu.

34. Notons toutefois que dans la "vraie vie", les choses ne furent pas aussi simples et aisées entre Lena et sa belle-famille, au point d'aboutir très vite à une rupture définitive.

35. À ce sujet, voir par exemple Bruno Cabanes, «La démobilisation des soldats français ", Les cahiers de la paix, $n^{\circ} 7$, Nancy, Presses de l'Université de Nancy, 2000, p. 55-65 ; Bruno Cabanes, La victoire endeuillée. La sortie de guerre des soldats français (1918-1920), Paris, Seuil, 2004 ; Guillaume Piketty et Vladimir Trouplin, Les compagnons de l'aube. Archives inédites des compagnons de la Libération, Paris, Éditions Textuel, 2014.

36. La future madame Leckie.

37. Aujourd'hui Auburn University.

38. Stephen E. Ambrose, Band of Brothers, E Company, 506th Regiment, 101st Airborne from Normandy to Hitler's Eagle's Nest, New York, Simon \& Schuster, 1992.

39. Robert Leckie, Helmet for my Pillow. Op. cit.

40. Eugene B. Sledge, With The Old Breed. Op. cit.. L'épisode 10 de la mini-série a été également fondé sur : Eugene B. Sledge, China Marine. An Infantryman's Life After World War Two, New York, Oxford University Press, 2003.

41. Chuck Tatum, Red Blood, Black Sand. Fighting alongside John Basilone from Boot Camp to Iwo Jima, New York, Berkley Trade, 2013.

42. Notamment Sydney Phillips, You'll Be Sor-ree! A Guadalcanal Marine Remembers the Pacific War, New York, Berkley Trade, 2012.

43. En particulier le journal de Tatum et des objets conservés par la famille de John Basilone.

44. Ainsi, l'historien Hugh Ambrose a rédigé un livre pendant qu'il travaillait à la mini-série (livre paru en 2010) : The Pacific. Hell was an Ocean Away, New York, New American Library, 2010.

45. Songeons par exemple aux photographies de combattants alliés qui ont émergé décharnés de la campagne victorieuse contre les Italiens en Érythrée (hiver 1941).

46. Pour « GIs and Marines ».

47. Au sens littéral du terme puisque le film / documentaire est notamment composé à partir des petits films tournés en Irak par les Marines avec leurs téléphones portables et autre minicaméras.

48. Telles que par exemple Le jour le plus long (1962), La grande évasion (1963), Tora! Tora! Tora! (1970), Patton (1970) ou encore La bataille de Midway (1976).

49. Tom Brokaw, Op. cit. Voir aussi Kenneth D. Rose, Myth and The Greatest Generation. A Social History of Americans in World War II, New York, Taylor and Francis Group, 2008.

50. Des combattants et... des téléspectateurs. 


\section{RÉSUMÉS}

Héritières de Saving Private Ryan (1998), les mini-séries Band of Brothers (2001) et The Pacific (2010) présentent la Seconde Guerre mondiale telle que l'expérimentèrent les hommes de la $101^{\text {ème }}$ division aéroportée américaine en Europe (Band of Brothers) et les US Marines face aux Japonais ( The Pacific). Conçues et réalisées selon des modalités semblables, elles se concentrent l'une et l'autre sur le champ de bataille et se dispensent de toute allusion aux enjeux diplomatiques, politiques, économiques et sociaux du conflit. Mais les similitudes s'arrêtent là. Si les parachutistes et les Marines sont décrits comme d'efficaces combattants, les premiers sont dépeints sous un jour plus flatteur que les seconds. Compétente et rarement battue en brèche dans Band of Brothers, la hiérarchie est régulièrement critiquée dans The Pacific. Les relations entre combattants et civils sont davantage mises en évidence dans la seconde série que dans la première. Par ailleurs, d'une œuvre à l'autre, les représentations de l'ennemi, du combat et de ses conséquences évoluent substantiellement. Si Band of Brothers décrit un soldat allemand redoutable dont la valeur souligne celle des parachutistes américains, The Pacific montre la dimension raciale de la guerre et les terribles difficultés posées par cet autre ennemi que fut l'environnement. Présentes sur les champs de bataille européens, la violence, la blessure et la mort sont plus crûment mises en scène sur le théâtre Pacifique. Enfin, les conséquences du conflit sur les combattants et le délicat processus de sortie de guerre sont incomparablement mieux évoqués dans The Pacific que dans Band of Brothers. Ces variations sont liées aux différences entre les sources sur lesquelles les mini-séries sont fondées, à la nature du phénomène guerrier que celles-ci présentent, aux changements de contexte historiographique et cinématographique, ainsi qu'aux évolutions des environnements politiques américain et international.

Heirs of Saving Private Ryan (1998), the mini-series Band of Brothers (2001) and The Pacific (2010) present the Second World War as is was experienced by the men of the $101^{\text {st }}$ US Airborne Division in Europe (Band of Brothers) and by the US Marines against the Japanese (The Pacific). Designed and directed under similar terms, they both focus on the battlefield. Thus they do not make any reference to the diplomatic, political, economic and social issues raised by the conflict. But the similarities end there. If the paratroopers and the Marines are described as effective combatants, the former are portrayed in a more flattering light than the latter. Competent and rarely undermined in Band of Brothers, the hierarchy is regularly criticized in The Pacific. The relationships between combatants and civilians are much more highlighted in the second miniseries than in the first. Moreover, from one series to the other, the representations of the enemy, of the fighting and of its consequences change substantially. If Band of Brothers describes a rather formidable German soldier whose value points out that of the American paratroopers, The Pacific shows the racial dimension of the war and the terrible difficulties created by the other enemy that the environment was. Present on the European battlefields, violence, injury and death are more crudely staged on the Pacific theatre. Finally, the consequences of the conflict on the combatants and the complex coming out of war process are incomparably better evoked in The Pacific than in Band of Brothers. These variations are related to the differences between the sources on which the mini-series are based, to the nature of the warlike phenomenon that they present, to the changes in terms of historiographical and film contexts, as well as to the evolutions of the US and international political environments. 


\section{INDEX}

Keywords : Spielberg Steven, US Marines, Second World War, home front, warlike phenomenon, enemy, violence, environment, coming out of war, Band of Brothers, Pacific (The)

Mots-clés : Spielberg Steven, US Marines, Seconde Guerre mondiale, phénomène guerrier, ennemi, violence, environnement, sortie de guerre, Band of Brothers, Pacific (The)

\section{AUTEUR}

\section{GUILLAUME PIKETTY}

Guillaume Piketty est Professeur d'histoire à Sciences Po (Paris) et Visiting Fellow au Worcester College (Oxford). Après une série d'études sur la Résistance intérieure française et sur la France libre, ses recherches portent aujourd'hui sur l'histoire sociale et culturelle de la Seconde Guerre mondiale en France et en Europe, et, plus largement, sur le phénomène guerrier et le phénomène résistant depuis la fin de la guerre civile américaine. Il met notamment l'accent sur l'expérience quotidienne des exilés européens durant la Seconde Guerre mondiale, ainsi que sur les liens entre guerre, sensibilités et émotions.

Guillaume Piketty is a full professor of History at Sciences Po (Paris) and a Visiting Fellow at Worcester College (Oxford). After a series of studies on the French Resistance and on Free France, he now works on the social and cultural history of the Second World War in France and in Europe, and, more broadly, on war and resistance since the end of the US Civil War. His research notably focuses on the day-to-day experience of the European exiles during the Second World War, and on the relationships between war, sensitivity and emotions. 